\title{
Fracture prevention service to bridge the osteoporosis care gap
}

This article was published in the following Dove Press journal:

Clinical Interventions in Aging

25 June 2015

Number of times this article has been viewed

\section{Carmelinda Ruggiero' \\ Elena Zampi ${ }^{1}$ \\ Giuseppe Rinonapoli ${ }^{2}$ \\ Marta Baroni' \\ Rocco Serra' \\ Elisa Zengarini' \\ Gregorio Baglioni ${ }^{3}$ \\ Giuliana Duranti ${ }^{3}$ \\ Sara Ercolani' \\ Francesco Conti ${ }^{4}$ \\ Auro Caraffa ${ }^{2}$ \\ Patrizia Mecocci' \\ Maria Luisa Brandi \\ 'Geriatric Unit, University of Perugia, Hospital S Maria della Misericordia, \\ ${ }^{2}$ Orthopedic and Traumatologic \\ Unit, University of Perugia, Hospital \\ S Maria della Misericordia, ${ }^{3}$ Primary \\ Care Physicians, SIMG Umbria, \\ Perugia, ${ }^{4}$ Department of Clinical and Molecular Medicine, Sapienza \\ University of Rome, AO Sant'Andrea \\ Hospital, Rome, ${ }^{5}$ Department of \\ Internal Medicine, University of \\ Florence, Florence, Italy}

Correspondence: Carmelinda Ruggiero Department of Medicine, Institute of Gerontology and Geriatrics, University of Perugia Medical School, S Maria della Misericordia Hospital, Sant'Andrea delle Fratte, 06100 Perugia, Italy

Tel +39075 5783839

Email carmelinda.ruggiero@unipg.it
Background: A care gap exists between the health care needs of older persons with fragility fractures and the therapeutic answers they receive. The Fracture Prevention Service (FPS), a tailored in-hospital model of care, may effectively bridge the osteoporosis care gap for hipfractured older persons. The purpose of this study was to evaluate the efficacy of the FPS in targeting persons at high risk of future fracture and to improve their adherence to treatment.

Methods: This was a prospective observational study conducted in a teaching hospital with traumatology and geriatric units, and had a pre-intervention and post-intervention phase. The records of 172 participants were evaluated in the pre-intervention phase, while data from 210 participants were gathered in the post-intervention phase. All participants underwent telephone follow-up at 12 months after hospital discharge. The participants were patients aged $\geq 65$ years admitted to the orthopedic acute ward who underwent surgical repair of a proximal femoral fracture. A multidisciplinary integrated model of care was established. Dedicated pathways were implemented in clinical practice to optimize the identification of high-risk persons, improve their evaluation through bone mineral density testing and blood examinations, and initiate an appropriate treatment for secondary prevention of falls and fragility fractures.

Results: Compared with the pre-intervention phase, more hip-fractured persons received bone mineral density testing ( $47.62 \%$ versus $14.53 \%, P<0.0001)$, specific pharmacological treatments $(48.51 \%$ versus $17.16 \%, P<0.0001)$, and an appointment for evaluation at a fall and fracture clinic $(52.48 \%$ versus $2.37 \%, P<0.0001)$ in the post-intervention phase. Independent of some confounders, implementation of the FPS was positively associated with recommendations for secondary fracture prevention at discharge $(P<0.0001)$ and with 1 -year adherence to pharmacological treatment $(P<0.0001)$.

Conclusion: The FPS is an effective multidisciplinary integrated model of care to optimize identification of older persons at highest risk for fragility fracture, to improve their clinical management, and to increase adherence to prescriptions.

Keywords: osteoporosis, secondary prevention, hip fracture, fracture liaison service, model of care

\section{Introduction}

Osteoporotic fractures mainly occur in older persons worldwide. In Italy, 90,000 hip fractures per year affect persons aged older than 50 years, with a growing trend coincident with aging of the population. ${ }^{1}$ In the USA, $90 \%$ of 350,000 hip fractures per year occur in persons over 65 years. $^{2}$ Western countries have generally reported increases in hip fracture incidence through the second half of the last century. Hip fracture rates have stabilized over the last 2 decades, with age-adjusted decreases being observed in some centers. ${ }^{3}$

A fragility fracture at the hip is devastating and is associated with increased morbidity, disability, and mortality. ${ }^{4}$ Epidemiological studies show an annual mortality 
rate over $25 \%$ and incomplete recovery of pre-fracture mobility in more than $50 \%$ of survivors. ${ }^{5-7}$ Approximately $60 \%$ of hip-fractured patients require assistance with activities of daily living, and $10 \%-20 \%$ of these patients are admitted to care homes in the year following the event. ${ }^{8}$ Hip fracture is also a strong risk factor for future vertebral and non-vertebral fractures. Patients who suffer a hip fracture are two to four times as likely to have a further hip fracture, with $10 \%$ having another fracture within 1 year. ${ }^{9}$ Approximately $35 \%$ of patients hospitalized for a fragility fracture have a higher risk of being readmitted, with a further fragility fracture within 1-2 years of the previous one. ${ }^{10}$ The economic burden associated with direct costs (including acute in-hospital treatment, post-operative complications, rehabilitation programs and the use of the health services) has been estimated to be $\$ 20$ billion in the USA and $€ 30$ billion in the European Union. An additional burden is attributable to indirect costs (including burden for family members and caregivers due to the onset or increased disabilities) of hip fractures that have been only partially evaluated and weigh heavily on health care systems. ${ }^{11,12}$ Although guideline-recommended osteoporosis treatments can dramatically reduce fracture risk by up to $50 \%$, about $50 \%$ of osteoporotic women and $90 \%$ of men with incident fragility fracture are not treated with any anti-fracture medication. ${ }^{13-17}$ Further, less than $25 \%$ of older patients who sustain fractures receive adequate vitamin D supplementation. ${ }^{18,19}$

In this scenario, fracture liaison services (FLS) have been developed in many health systems to identify persons at high risk for fragility fractures and/or to encourage outpatient primary care physicians to initiate appropriate treatment. ${ }^{20-22}$ Epidemiological studies shown that these services may narrow the care gap for patients with fragility fractures, and they are clinically effective and cost-effective in providing secondary preventive care. ${ }^{23}$ Since FLS have not been widely adopted, mainly due to staff and service reorganization, no improvement in the osteoporotic care gap has been identified in recent years. ${ }^{24,25}$ The Fracture Prevention Service (FPS) is a multidisciplinary model of care tailored to identify, assess, and treat in an effective and timely manner all patients who suffer a hip fracture and are at high risk of subsequent fracture. In our experience, FPS was developed by optimizing available resources through a structured path that includes involvement of general practitioners, orthopedic surgeons, and other specialists involved in the treatment of osteoporosis.

The aim of this study was to evaluate the feasibility of the FPS and to estimate the association between implementation of the FPS and outcome measures related to secondary prevention of fragility fractures, including bone mineral density (BMD) testing, evaluation at a fall and fracture clinic, drug prescription, and adherence to pharmacological treatment. The FPS was adapted to address the needs of specific patient populations and it resembles the model of FLS that is currently considered to be the most effective for management of hip-fractured persons. ${ }^{26}$ We believe that given the importance of hip fracture to the Italian health system and its impact on the quality of life of elderly people, building an FPS as in other countries is a mandatory objective.

\section{Materials and methods Study design and sample}

The FPS is a multidisciplinary and collaborative team, including orthopedic surgeons, geriatricians, physical therapists, nurses, and general practitioners, which assumes responsibility for secondary fracture prevention. The FPS assures case finding, assessment, and diagnostic evaluation, and encourages outpatient primary care physicians to initiate appropriate treatment. During the period AprilDecember 2011, geriatricians, as providers of a fall and fracture clinic, met with orthopedic surgeons, nurses, physical therapists, and primary care physicians to determine the needs and preferences of these groups for collaboration in order to assure the secondary prevention of fragility fractures. The fall and fracture clinic is a hospital-based outpatient ambulatory service provided jointly by a geriatrician and a physiotherapist. Teaching and training activities were undertaken for staff involved in the FPS to raise awareness concerning guidelines for the diagnosis and treatment of osteoporosis. A fragility fracture protocol was established, and written guidelines for assessment and treatment of persons with a proximal hip fracture were defined for staff in the orthopedic and traumatology department. In detail, low trauma hip fracture is defined as a fracture sustained as a result of a fall from standing height or less and not occurring as a consequence of a road traffic accident. All persons aged 65 years and older admitted with low trauma hip fracture are identified by the orthopedic surgeons and sent the fragility fracture protocol. The protocol includes BMD and blood testing, assessment of fracture risk, and prescription and monitoring of antiosteoporotic treatment. At discharge, the FPS team members send a letter to the general practitioner explaining that their patient is scheduled for a comprehensive fracture and falls risk assessment, including BMD evaluation and blood tests. The main reason for a FPS in our teaching hospital is the large number of surgical procedures done for 
proximal femoral fracture ( $\mathrm{n}=700$ in 2010) in the orthopedics and traumatology department.

In this study, we examined the data gathered from patients with proximal hip fracture admitted to the orthopedics and traumatology department of the teaching hospital at Perugia from October 2010 to March 2011 and from January to June 2012, respectively, before and after implementation of the multidisciplinary and collaborative FPS team. For the purposes of the study, all participants received 6-month and 12-month telephone follow-up by two trained nurses. The study protocol complied with the Declaration of Helsinki and was approved by the ethics committee of the regional health care system.

\section{Demographic, clinical, and treatment- related characteristics}

We collected demographic data and information concerning the type of surgery used to treat the hip fracture, along with the type and site of previous fragility fractures, by reviewing the clinical charts of patients with proximal hip fracture. The type of hip fracture was classified as femoral neck, subtrochanteric, periprosthetic, or subtrochanteric. Previous fragility fracture sites were classified as hip, vertebral, wrist, and other. Information was collected on instrumental evaluation, based on BMD testing and drug therapy for osteoporosis, ie, calcium \pm vitamin D supplementation and bisphosphonates or other agents ongoing before admission and/or recommended at discharge from the orthopedic ward. In addition, first level blood testing for secondary osteoporosis, including calcium, phosphorus, and plasma intact parathyroid hormone were collected when evaluated during the hospital stay. All participants received telephone follow-up with the aim of obtaining information about drug adherence or, where appropriate reasons for suspension, occurrence of falls, fractures, major clinical events requiring hospitalization, and/or mortality. Participants' vital status was determined by telephone follow-up, correspondence, and searches of the Regional Death Index (updated in December 2013).

\section{Statistical analysis}

Participants' characteristics were described according to their pre-intervention (group A) or post-intervention (group B) enrollment status. Continuous variables with a symmetric distribution were reported as the mean and standard deviation, while those with an asymmetric distribution were summarized as the median and interquartile range. Categorical variables were summarized as proportions and percentages.
Table I Multivariate analysis of the association between activation of the Fracture Prevention Service and pharmacological treatment for secondary prevention of fragility fractures at hospital discharge

\begin{tabular}{llll}
\hline & \multicolumn{3}{l}{$\begin{array}{l}\text { Pharmacological secondary } \\
\text { prevention }\end{array}$} \\
\cline { 2 - 4 } & $\boldsymbol{\beta}$ & SE & P-value \\
\hline Fracture unit & 0.34 & 0.04 & $<0.000 \mathrm{I}$ \\
Age & $-0.00 \mathrm{I}$ & 0.002 & 0.6875 \\
Female sex & 0.14 & 0.05 & 0.0044 \\
Specific treatment at admission & 0.44 & 0.07 & $<0.000 \mathrm{I}$ \\
History of fracture & -0.13 & 0.04 & $0.005 \mathrm{I}$ \\
\hline
\end{tabular}

Abbreviation: SE, standard error.

Statistical comparisons across groups were performed using the Fisher's Exact test, $t$-test, or chi-square test, as appropriate, and sensitivity analysis was performed using McNemar's test. The significance level used for two-sided tests was $P<0.05$. The probability of patients receiving a specific antifracture drug prescription at hospital discharge as well as their 1-year adherence to prescribed drugs were compared post-FPS versus pre-FPS using multivariate regression based on a generalized linear model procedure (Tables 1 and 2, respectively). Parsimonious models were identified using the stepwise selection method within "proc reg" procedures. All analyses were performed using SAS software version 9.3 (SAS Institute, Cary, NC, USA).

\section{Results}

Table 3 shows the characteristics of the participants grouped according to their enrollment phase, ie, before (group A) or after (group B) activation of the FPS, and stratified according to the time of evaluation during their hospital stay, ie, at hospital admission or discharge. Overall, 382 hip-fractured older persons were enrolled in the study, comprising 172 in the 6 months before (group A) and 210 in the 6 months after

Table 2 Multivariate analysis of the association between implementation of the Fracture Prevention Service and I-year adherence to pharmacological treatment for secondary prevention of fragility fracture

\begin{tabular}{llll}
\hline & \multicolumn{2}{l}{$\begin{array}{l}\text { One-year adherence to secondary } \\
\text { prevention }\end{array}$} & \\
\cline { 2 - 4 } & $\boldsymbol{\beta}$ & SE & P-value \\
\hline Fracture unit & 0.19 & 0.03 & $<0.0001$ \\
Age & -0.001 & 0.002 & 0.4864 \\
Female sex & 0.09 & 0.03 & 0.0154 \\
\hline
\end{tabular}

Notes: Further confounders, including history of fracture, type of fracture, destination at discharge, history of specific treatment, and calcium and/or vitamin D supplementation at hospital admission were tested and excluded from the parsimonious model using stepwise regression method analysis.

Abbreviation: SE, standard error. 
Table 3 Characteristics of participants enrolled before (group A) and after (group B) activation of the Fracture Prevention Service according to time of hospital assessment (at admission and at discharge, respectively)

\begin{tabular}{|c|c|c|c|}
\hline & $\begin{array}{l}\text { Group A } \\
(n=172)\end{array}$ & $\begin{array}{l}\text { Group B } \\
(n=2 \mid 0)\end{array}$ & $P$-value \\
\hline Women, n (\%) & I 35 (78.49) & I5I (7I.90) & 0.1400 \\
\hline Age, years, mean $\pm S D$ & $83.32 \pm 8.16$ & $83.52 \pm 7.47$ & 0.8090 \\
\hline \multicolumn{4}{|l|}{ Clinical characteristics } \\
\hline Type of fracture, n (\%) & & & 0.4488 \\
\hline Femoral neck & $85(49.42)$ & $89(42.38)$ & \\
\hline - Trochanteric & $79(45.93)$ & $113(53.81)$ & \\
\hline - Periprosthetic & $2(1.16)$ & $3(1.43)$ & \\
\hline - Subtrochanteric & $6(3.49)$ & $5(2.38)$ & \\
\hline Type of surgery, $n(\%)$ & & & 0.6178 \\
\hline - Osteosynthesis & $103(59.88)$ & $137(64.76)$ & \\
\hline - Prosthesis & $68(39.53)$ & $73(34.76)$ & \\
\hline Previous fracture, $\mathrm{n}(\%)$ & $64(37.21)$ & $75(35.71)$ & 0.7625 \\
\hline Previous fracture site, $\mathrm{n}(\%)$ & & & 0.1210 \\
\hline - Femur & $34(53.97)$ & $27(36.00)$ & \\
\hline - Vertebra & $8(12.70)$ & $12(16.00)$ & \\
\hline - Wrist & $9(14.29)$ & $10(13.33)$ & \\
\hline - Other & $12(19.05)$ & $26(34.66)$ & \\
\hline \multicolumn{4}{|l|}{ Diagnostic and therapy before index fracture } \\
\hline BMD testing, $n(\%)$ & $3(1.74)$ & I (0.48) & 0.2258 \\
\hline Antiosteoporosis drug treatment, $\mathrm{n}(\%)$ & $18(10.47)$ & $15(7.14)$ & 0.2502 \\
\hline Calcium and/or vitamin D supplementation, $\mathrm{n}(\%)$ & $19(11.05)$ & II (5.24) & 0.0358 \\
\hline \multicolumn{4}{|l|}{ Diagnostic and therapy after index fracture } \\
\hline BMD testing, n (\%) & $25(14.53)$ & $100(47.62)$ & $<0.000$ I \\
\hline Fall and fractures clinic evaluation, $n(\%)$ & $4(2.37)$ & $106(52.48)$ & $<0.000$ I \\
\hline Antiosteoporosis drug treatment, $\mathrm{n}(\%)$ & $29(17.16)$ & $98(48.5 \mathrm{I})$ & $<0.000$ I \\
\hline Calcium/vitamin D supplementation, $\mathrm{n}(\%)$ & $28(16.57)$ & $101(50.00)$ & $<0.000$ I \\
\hline
\end{tabular}

Note: $P$-value from Fisher's Exact test, $t$-test, or chi-square test, as appropriate.

Abbreviations: BMD, bone mineral density; SD, standard deviation.

(group B) activation of the FPS. The majority of participants were women $(78.49 \%$ versus $71.90 \%, P=0.1400)$ and aged over 80 years $(83.32 \pm 8.16$ versus $83.52 \pm 7.47, P=0.8090)$. In both groups, the most prevalent proximal femoral fractures were at the femoral neck ( $49.42 \%$ versus $42.38 \%$, respectively) and subtrochanteric (45.93\% versus $53.81 \%)$ sites. Sixty percent of hip-fractured patients underwent an osteosynthesis procedure (59.88\% versus $64.76 \%$ in the preintervention group and post-intervention group, respectively) and about one-third underwent a prosthetic replacement, ie, total arthroplasty or bipolar hip prosthesis $(39.53 \%$ versus $34.76 \%$ ). Overall, one of every three patients had already experienced a fragility fracture, with the hip being the most prevalent site $(53.97 \%$ versus $36.00 \%)$, followed by the wrist $(14.29 \%$ versus $13.33 \%)$ and vertebrae $(12.70 \%$ versus $16.00 \%$ ). Less than $10 \%$ of patients were on antiosteoporosis drug treatment $(10.47 \%$ versus $7.14 \%, P=0.2502)$ and/or vitamin $\mathrm{D} \pm$ calcium supplementation $(11.05 \%$ versus $5.24 \%, P=0.0358)$ at admission. Few persons $(1.74 \%$ versus $0.48 \%$ ) reported having BMD testing in the years before their fracture. Compared with group A, participants enrolled after activation of the FPS (group B) had a higher probability of receiving recommendations for secondary prevention of fracture at discharge. About half of them were prescribed specific drug treatment $(48.51 \%$ versus $17.16 \%, P<0.0001)$ and calcium \pm vitamin $\mathrm{D}$ supplementation $(50.00 \%$ versus $16.57 \%, P<0.0001)$, and obtained indications for BMD testing (47.62\% versus $14.53 \%, P<0.0001)$ and evaluation of fall and fracture risk (52.48\% versus $2.37 \%, P<0.0001)$ within 3 months of discharge. Bisphosphonates were the most commonly prescribed drugs for secondary fracture prevention at discharge. Almost all hip-fractured patients in group B (98.00\%) received calcium and vitamin D supplementation at discharge compared with $66.66 \%$ of those in group A. Almost all the characteristics of participants discharged after activation of the FPS were significantly different from those of patients discharged before the intervention (Table 3). Further, activation of the FPS was associated with the prescription of a complete treatment for secondary fracture prevention at hospital discharge, ie, a specific drug plus calcium and vitamin $\mathrm{D}$ supplementation, independent of age, sex, history of fracture, and ongoing drug treatment at admission 
Table 4 One-year adherence to recommendations prescribed at discharge among participants enrolled before (group A) and after (group B) implementation of the Fracture Prevention Service

\begin{tabular}{llll}
\hline & Group A & Group B & P-value \\
\hline Participants contributing to baseline, $\mathrm{n}(\%)$ & $172(100 \%)$ & $210(100 \%)$ & - \\
One-year mortality, $\mathrm{n}(\%)$ & $22(\mathrm{I} 2.7)$ & $33(15.7)$ & $0.50 \mathrm{II}$ \\
One-year dropout, $\mathrm{n}(\%)$ & $34(19.76)$ & $35(16.66)$ & 0.4332 \\
Participants contributing to & $116(67.44)$ & $142(67.61)$ & 0.1655 \\
I-year follow-up, $\mathrm{n}(\%)$ & & & \\
BMD testing, $\mathrm{n}(\%)$ & $24(20.68)$ & $62(43.66)$ & $<0.000 \mathrm{I}$ \\
Fall and fracture clinic attendance, $\mathrm{n}(\%)$ & $6(5.17)$ & $78(54.92)$ & $<0.000 \mathrm{I}$ \\
Specific antifracture drug therapy, $\mathrm{n}(\%)$ & $17(14.65)$ & $56(39.43)$ & $<0.000 \mathrm{I}$ \\
Calcium plus vitamin D supplementation, $\mathrm{n}(\%)$ & $10(8.62)$ & $65(45.77)$ & $<0.000 \mathrm{I}$ \\
\hline
\end{tabular}

Note: $P$-value from Fisher's Exact test, $t$-test, or chi-square test, as appropriate. Abbreviation: BMD, bone mineral density.

(Table 1). No interaction term between antifracture treatment at admission and FPS was found in the model. Table 4 shows 1 -year adherence to prescriptions given to patients at hospital discharge and still active in follow-up ( $n=116$ [67.44\%] of those enrolled in group $A$ and $n=142$ [67.61\%] of those enrolled in group B). Of note, $15.7 \%(n=33)$ and $12.7 \%$ $(\mathrm{n}=22)$ of the participants died within 1 year of follow-up, while $16.6 \%(n=35)$ and $19.7 \%(n=34)$ dropped out for other reasons from group B (post-intervention) and group A (preintervention), respectively.

Of note, $15.7 \%(n=33)$ and $12.7 \%(n=22)$ of the participants died within the 1-year follow-up, while $16.6 \%(n=35)$ and $19.7 \%(n=34)$ dropped out for other reasons from group B (post-intervention) and group A (pre-intervention), respectively.

Compared with patients in group A, those enrolled after FPS activation (group B) had higher adherence to BMD testing (19.64\% versus $50.43 \%, P<0.0001)$, fall and fracture clinic evaluation $(4.50 \%$ versus $64.41 \%, P<0.0001)$, and persisted on antiosteoporosis drugs (14.04\% versus $44.07 \%$, $P<0.0001)$ and calcium plus vitamin D supplementation (8.26\% versus $53.51 \%, P<0.0001$, Table 4$)$. Independent of age, sex, history of fracture, and ongoing drug treatments at admission, activation of the FPS was positively associated with 1-year adherence to complete pharmacological treatment, ie, an antiosteoporosis drug plus vitamin $\mathrm{D}$ and calcium supplementation, for secondary prevention of fragility fractures (Table 2). No interaction term was found between activation of the FPS and history of specific treatment.

\section{Discussion}

Activation of the FPS, a multidisciplinary integrated model of care for secondary prevention of fragility fractures, is an effective strategy for increasing the probability that older adults at the highest risk of a subsequent fragility fracture receive BMD testing, fall and fracture risk assessment, antifracture medications, and for improving 1-year adherence to treatment. In our experience, the FPS resembles a coordinator-based system with an osteoporosis care manager, ie, a geriatrician or orthopedic surgeon, to explain the need for a secondary prevention fracture program to older persons, their relatives, and/or general practitioner, and with a dedicated nurse available to contact and organize access to the outpatient clinic for completing the diagnostic and therapeutic pathway.

Several models of FLS have been developed depending on the setting, target population, health care providers, and the characteristics of specific health care systems..$^{27,28}$ Direct comparisons among models of FLS are not feasible because they were developed according to a different health care system organization depending on the countries. In fact, drawing conclusions on the efficacy of a given model should be done considering the existing resources, the actual local organization, and the priorities of the health care system. Compared with previous studies, we confirmed a trend toward a higher success rate of coordinated systems for secondary prevention in hip fracture patients. Although the design of the study was an observational pre-/post-intervention, the rates of therapeutic and diagnostic antifracture recommendations markedly increased at discharge and were of similar magnitude to those in previous randomized controlled trials ${ }^{29-31}$ and studies with a similar design. ${ }^{32,33}$ At hospital discharge, one in two of our patients received appropriate management, including BMD testing, fall and fracture risk evaluation, and antifracture drug treatment. In addition, we showed significantly increased adherence 1 year after the hip fracture: Fifty-two percent of the participants still active in follow-up attended the fall and fracture clinic, $43 \%$ did the BMD testing, and approximately $40 \%$ were on specific antifracture drug treatment. We acknowledge that still a large proportion of participants 
desire indications for antifracture drug treatments, both at discharge as well as 1-year after fracture. Several factors are involved in the processes associated with improvement of such outcomes; some are related to the organizational aspects of the multidisciplinary team, but others are due to a limited understanding of the deleterious consequences of fractures on the part of patients and their carers. Several lines of evidence suggest that bone-protecting treatments reduce adverse outcomes and the incidence of subsequent fracture without prior dual-energy X-ray absorptiometry scanning, ${ }^{15}$ although there is some controversy about the need for BMD scanning before starting secondary prevention.

An important aspect of this study is that it describes the first coordinator-based program to include an integrated approach for fall and fracture prevention in more than one half of participants attending an outpatient clinic. However, the effects of such a fall prevention program on fracture risk still needs to be clarified in this study sample. Another key element contributing to the success of this program is that it promoted a high level of adherence to medications by proactively monitoring patients over time. The improvement of 1-year drug adherence in our study is an unique finding that was not investigated in previous randomized trials, nor in observational studies with a similar design. In addition, our model of FPS was designed to capture, evaluate, and treat the highest fracture risk stratum of the population. Hipfractured patients are particularly difficult to bring back to the FPS because of mobility and frailty issues. In our study, the FPS was structured as a multidisciplinary path, integrating available services and specialists within the hospital, to whom hip-fractured patients are automatically referred at the time they are hospitalized. Thus, activation of an appropriate secondary fracture prevention protocol would no longer depend exclusively on the attending physician in the surgical (orthopedic) department, nor the patients should be more committed to looking for secondary fracture prevention after hospital discharge. Ultimately, a key factor in maximizing the beneficial effects of FPS was the involvement of the primary care physicians who contribute to begin therapy as well as monitor the continuity of care. Poor adherence with medications for chronic diseases is a common problem in many patients, especially among older persons with inadequate understanding of their diseases and the detrimental impact on the functionality. Involvement of geriatricians in the FPS overcomes several issues related to choice of treatment, management of comorbidities such as cognitive disorders, depression and chronic disease, polypharmacy, patient's life expectancy, and preference. Coordinated interaction of the different professional disciplines involved, ie, geriatrician, orthopedic surgeon, nurse specialist, physiatrist, and general practitioner, and better comprehension of the risks associated with subsequent fragility fractures on the part of patients and their cares, might have contributed to the improved adherence to pharmacological treatment seen in our study. The good compliance with antifracture treatment (more than 50\% in 1 year) may also be ascribed to a more tailored choice of antifracture drugs. This may be considered a good result and represents an important and encouraging outcome of such intervention. Ultimately, the communication and the comprehension of the issues associated with fragility fractures improved among the older adults and their caregivers. Possible reasons for the higher compliance with antifracture treatment after activation of the FPS might be ascribed to a more tailored choice of antifracture drugs and improved comprehension of the risks associated with subsequent fragility fractures.

Several studies from Australia, Canada, France, Ireland, Spain, the Netherlands, the UK, and the USA have described the structure and performance of FLS protocols. The optimal way to organize and deliver an FLS has been the subject of ongoing debate among service leaders and the focus of several systematic reviews. ${ }^{20-22,27,28,34-40}$ The most recent review suggests that only type $\mathrm{A}$, ie, an all-encompassing service where patients with fragility fracture are identified, assessed, and treated for osteoporosis with a fracture liaison coordinator being central to the service, has demonstrated a significant reduction on the incidence of refracture. Our findings are consistent with these reports. ${ }^{25}$

Availability of a centralized database and a tracking system helps provide the appropriate diagnostic and therapeutic protocol. Therefore, funding the information technology area might ultimately be cost-saving. However, in our experience, competing priorities for nursing time led to suboptimal data entry and difficulties in patients tracking, confirming the importance of identifying dedicated staff. The main problems are not medical but logistic, and the answers to these issues may lie in organizational aspects. Overcoming these issues is a priority since the spread of systematic refracture prevention programs in Italy and elsewhere should be given priority.

We acknowledge that our study has some limitations. Our evaluation of long-term adherence to antifracture drug prescriptions and diagnostic recommendations is limited to $67 \%$ of the original sample, mainly because of the high mortality rate $(13 \%-16 \%)$ and dropout rate $(12 \%-15 \%)$ in the study population. The main reasons for dropout were change of address, unwillingness to be interviewed, deterioration of health status, and onset of severe disability. 
Further, our participants with hip fracture did not undergo a comprehensive geriatric evaluation during their hospital stay in the orthopedic ward. Therefore, clinical and functionrelated variables that may affect the outcomes of FPS were not included in the analyses. Ultimately, this is a "before and after" study that relies on historical controls (before and after the period that the FPS was in effect), and not a randomized controlled trial. Because this study simply observed a change in the number of participants that received specific antifracture recommendations, there is always the chance that the change we observed was due to some unobserved confounding variable or systematic bias.

In conclusion, the FPS program improved the care of patients with fragility fractures and positively impacted the cultural communication and education of health care providers. By identifying and addressing the needs of older adults at highest risk for fragility fractures, the FPS is expected to have long-term health and economic benefits for both patients and the community. We believe that development of the FPS is effective for narrowing the deficit of care that exists in the management of patients with fragility fractures, and it is a priority for health care systems worldwide.

\section{Acknowledgments}

The authors would like to thank all patients and staff in the geriatric and traumatology units at S Maria della Misericordia Hospital. The study was entirely sponsored by these geriatric and traumatology units, and was not supported by external funding. The preliminary findings of this study were presented as a poster at the Ninth European Geriatric Medicine Society Congress and subsequently published in abstract form. ${ }^{40}$

\section{Author contributions}

All authors contributed to the conception and design of the study, acquisition of medical data, analysis and interpretation of data, drafting of the manuscript. All authors read and approved the final paper.

\section{Disclosure}

The authors report no conflicts of interest in this work.

\section{References}

1. Piscitelli P, Iolascon G, Argentiero A. Incidence and costs of hip fractures vs strokes and acute myocardial infarction in Italy: comparative analysis based on national hospitalization records. Clin Interv Aging. 2012;7:575-583.

2. Morris AH, Zuckerman JD. National consensus conference on improving the continuum of care for patients with hip fracture. J Bone Joint Surg Am. 2002;84:670-674.
3. Cooper C, Cole ZA, Holroyd CR, et al. IOF CSA Working Group on Fracture Epidemiology. Secular trends in the incidence of hip and other osteoporotic fractures. Osteoporos Int. 2011;22:1277-1288.

4. Maggi S, Siviero P, Wetle T, Besdine RW, Saugo M, Crepaldi G. A multicenter survey on profile of care for hip fracture: predictors of mortality and disability. Osteoporos Int. 2010;21:223-231.

5. Abrahamsen B, van Staa T, Ariely R, Olson M, Cooper C. Excess mortality following hip fracture: a systematic epidemiological review. Osteoporos Int. 2009;20:1633-1650.

6. Sattui SE, Saag KG. Fracture mortality: associations with epidemiology and osteoporosis treatment. Nat Rev Endocrinol. 2014;10: $592-602$.

7. Brewer LM, Kelly R, Donegan C, Moore AR, Williams D. Poor return of functional mobility after hip fracture in older patients - it's time to improve on hip fracture prevention. J Am Geriatr Soc. 2011;59: $1562-1563$.

8. Beaupre LA, Jones CA, Johnston DW, Wilson DM, Majumdar SR. Recovery of function following a hip fracture in geriatric ambulatory persons living in nursing homes: prospective cohort study. J Am Geriatr Soc. 2012;60:1268-1273.

9. Center JR, Bliuc D, Nguyen TV, Eisman JA. Risk of subsequent fracture after low trauma fracture in men and women. JAMA. 2007;297: 387-394.

10. New South Wales Department of Health, Agency for Clinical Innovation, Musculoskeletal Network. New South Wales re-fracture admission data 2002-2008. Sydney, Australia: Agency for Clinical Innovation, 2010. Available from: http://www.health.nsw.gov.au/resources/gmct/ musculoskeletal/refracture_data_analysis_2009_pdf.asp. Accessed January 20, 2014.

11. Rossini M, Piscitelli P, Fitto F, et al. Incidence and socioeconomic burden of hip fractures in Italy. Reumatismo. 2005;57:97-102. Italian.

12. Budhia S, Mikyas Y, Tang M, Badamgarav E. Osteoporotic fractures: a systematic review of U.S. healthcare costs and resource utilization. Pharmacoeconomics. 2012;30:147-170.

13. National Osteoporosis Foundation. Clinician's Guide to Prevention and Treatment of Osteoporosis. Available from: http://www.nof. org/professionals/NOF_Clinicians_Guide.pdf. Accessed June 20, 2014.

14. Kanis JA, McCloskey EV, Johansson H, et al. Scientific Advisory Board of the European Society for Clinical and Economic Aspects of Osteoporosis and Osteoarthritis (ESCEO) and the Committee of Scientific Advisors of the International Osteoporosis Foundation (IOF). European guidance for the diagnosis and management of osteoporosis in postmenopausal women. Osteoporos Int. 2013;24:23-57.

15. Lyles KW, Colon-Emeric CS, Magaziner JS, et al. Zoledronic acid in reducing clinical fracture and mortality after hip fracture. $N$ Engl J Med. 2007;357:1799-1809.

16. Fraser LA, Ioannidis G, Adachi JD, et al. Fragility fractures and the osteoporosis care gap in women: the Canadian Multicentre Osteoporosis Study. Osteoporos Int. 2011;22:789-796.

17. Lih A, Nandapalan H, Kim M, et al. Targeted intervention reduces refracture rates in patients with incident non-vertebral osteoporotic fractures: a 4-year prospective controlled study. Osteoporos Int. 2011;22: $849-858$.

18. Leytin V, Beaudoin FL. Reducing hip fractures in the elderly. Clin Interv Aging. 2011;6:61-65.

19. Avenell A, Gillespie WJ, Gillespie LD, O'Connell DL. Vitamin D and vitamin $\mathrm{D}$ analogues for preventing fractures associated with involutional and post-menopausal osteoporosis. Cochrane Database Syst Rev. 2009;2:CD000227.

20. Ahmed M, Durcan L, O'Beirne J, Quinlan J, Pillay I. Fracture liaison service in a non-regional orthopaedic clinic - a cost-effective service. Ir Med J. 2012;105:24:26-27.

21. Falchetti A, Amedei L, Masi F, et al. Fracture unit: a (possible) model of implementation in Italy. Clin Cases Miner Bone Metab. 2011;8:9-12.

22. Mitchell PJ. Fracture Liaison Services: the UK experience. Osteoporos Int. 2011;3:487-494. 
23. McLellan AR, Wolowacz SE, Zimovetz EA, et al. Fracture liaison services for the evaluation and management of patients with osteoporotic fracture: a cost effectiveness evaluation based on data collected over 8 years of service provision. Osteoporos Int. 2011;22:2083-2098.

24. Giangregorio L, Papaioannou A, Cranney A, Zytaruk N, Adachi JD. Fragility fractures and the osteoporosis care gap: an international phenomenon. Semin Arthritis Rheum. 2006;35:293-305.

25. Leslie WD, Giangregorio LM, Yogendran M, et al. A population-based analysis of the post-fracture care gap 1996-2008: the situation is not improving. Osteoporos Int. 2012;23:1623-1629.

26. Ganda K, Puech M, Chen JS, et al. Models of care for the secondary prevention of osteoporotic fractures: a systematic review and metaanalysis. Osteoporos Int. 2013;24:393-406.

27. Akesson K, Marsh D, Mitchell PJ, et al. IOF Fracture Working Group. Capture the fracture: a best practice framework and global campaign to break the fragility fracture cycle. Osteoporos Int. 2013;24:2135-2152.

28. Marsh D, Akesson K, Beaton DE, et al. IOF CSA Fracture Working Group. Coordinator-based systems for secondary prevention in fragility fracture patients. Osteoporos Int. 2011;22:2051-2065.

29. Majumdar SR, Beaupre LA, Harley CH, et al. Use of a case manager to improve osteoporosis treatment after hip fracture - results of a randomized controlled trial. Arch Intern Med. 2007;167:2110-2115.

30. Davis JC, Ashe MC, Guy P, Khan KM. HipWatch: osteoporosis investigation and treatment after a hip fracture: a 6-month randomized controlled trial. J Gerontol A Biol Sci Med Sci. 2007;62:888-891.

31. Gardner MJ, Brophy RH, Demetrakopoulos D, et al. Interventions to improve osteoporosis treatment following hip fracture - a prospective, randomized trial. J Bone Joint Surg Am. 2005;87:3-7.
32. Jones G, Warr S, Francis E, Greenaway T. The effect of a fracture protocol on hospital prescriptions after minimal trauma fractured neck of the femur: a retrospective audit. Osteoporos Int. 2005;16:1277-1280.

33. Sidwell AI, Wilkinson TJ, Hanger HC. Secondary prevention of fractures in older people: evaluation of a protocol for the investigation and treatment of osteoporosis. Intern Med J. 2004;34:129-132.

34. Sivakumar BS, McDermott LM, Bell JJ, Pulle CR, Jayamaha S, Ottley MC. Dedicated hip fracture service: implementing a novel model of care. ANZ J Surg. 2013;83:559-563.

35. Boudou L, Gerbay B, Chopin F, Ollagnier E, Collet P, Thomas T. Management of osteoporosis in fracture liaison service associated with longterm adherence to treatment. Osteoporos Int. 2011;22:2099-2106.

36. Carpintero P, Gil-Garay E, Hernández-Vaquero D, Ferrer H, Munuera L. Interventions to improve inpatient osteoporosis management following first osteoporotic fracture: the PREVENT project. Arch Orthop Trauma Surg. 2009; 129:245-250.

37. Panneman MJ, Lips P, Sen SS, Herings RM. Under-treatment with anti-osteoporotic drugs after hospitalization for fracture. Osteoporos Int. 2004;15:120-124.

38. Dell R. Fracture prevention in Kaiser Permanente Southern California. Osteoporos Int. 2011;22:457-460.

39. Sale JE, Beaton D, Posen J, Elliot-Gibson V, Bogoch E. Systematic review on interventions to improve osteoporosis investigation and treatment in fragility fracture patients. Osteoporos Int. 2011;22: 2067-2082.

40. Michel JP, editor. 9th Congress European Geriatric Medicine; 2013 Oct 2-4; Venice Italy; Elsevier editor: London; 2013.
Clinical Interventions in Aging

\section{Publish your work in this journal}

Clinical Interventions in Aging is an international, peer-reviewed journal focusing on evidence-based reports on the value or lack thereof of treatments intended to prevent or delay the onset of maladaptive correlates of aging in human beings. This journal is indexed on PubMed Central, MedLine,

\section{Dovepress}

CAS, Scopus and the Elsevier Bibliographic databases. The manuscript management system is completely online and includes a very quick and fair peer-review system, which is all easy to use. Visit http://www.dovepress. com/testimonials.php to read real quotes from published authors. 